\title{
Human Resource Development in Asia - Thriving on dynamism \& change: Reflections from 2006 Asian HRD conference
}

\begin{abstract}
This article discusses the experiences of the conference management team and the host (Universiti Putra Malaysia -UPM) of the Fifth Asian Conference of the Academy of Human Resource Development, held in Putrajaya, Malaysia from 2 to 5 December 2006. In reviewing the conference, the following sub-topics are used for organizing the contents of the article: HRD in Malaysia; conference theme \& overview; participations/country representations and paper streams; keynote addresses; conference assessment; and conclusions. At the end, brief perspectives of the next Asian HRD conference to be held in China are also provided.
\end{abstract}

Keyword: Asian HRD, Malaysia, AHRD-Asia Conference 2006 